\title{
Comportamento Reológico de Farinha Mista Extrusada de Bagaço de Jabuticaba e Arroz
}

Diego P. R. Ascheri, Pedro H. X. F. Silva, José L. R. Ascheri \& Carlos W. P. de Carvalho

Avaliou-se o comportamento reológico de farinha extrusada de bagaço de jabuticaba e arroz à $25-50^{\circ} \mathrm{C}$. Os dados experimentais foram ajustados pelos modelos de Newton, Oswald-de-Walle e Herschel-Bulkley (H-B) na faixa de deformação de 2,64-66,0 s-1. H-B foi o que ajustou adequadamente os dados experimentais verificando o comportamento pseudoplástico das amostras. O efeito da temperatura sobre o índice de consistência de H-B foi representado por uma equação linear e o índice de comportamento de fluxo por uma equação do tipo Arrhenius, aumentando o índice de consistência com o aumento da temperatura e diminuindo o índice de comportamento de fluxo com o aquecimento.

Palavras-chave: reologia; extrusão termoplástica; farinha extrusada.

In this study was evaluated the rheological performance of extruded flour from blends of jabuticaba/rice at $25-50^{\circ} \mathrm{C}$. The experimental data were adjusted by Newton, Oswald-of-Walle and Herschel-Bulkley (HB) models in the deformation range of 2.64$66.0 \mathrm{~s}-1$. Experimental data HB was adjusted appropriately checking as pseudo plastic behavior. The temperature effect on the consistency index of HB was represented by a linear equation and the index flow behavior the Arrhenius equation type, increasing the rate of consistency with increasing temperature and decreasing the rate of flow with the behavior of warming.

Keywords: rheology; thermoplastic extrusion; extruded flour. 


\section{Introdução}

A jabuticaba apresenta reconhecido valor nutricional. Atualmente vem sendo explorada para a produção de Javine análogo ao vinho de uva ${ }^{1,2}$, no entanto, subprodutos vêm sendo gerados e pouco aproveitados. O bagaço gerado é rico em fibras e minerais e possui considerável teor protéico que pode ser aproveitado em farinhas mistas extrusadas (FME) para o preparo de produtos de panificação ${ }^{3}$. No entanto, o processo de extrusão desnatura proteínas e degrada amidos conferindo à FME propriedades físicas diferentes às do material nativo e por isso há necessidade de estudá-las, principalmente quanto a seu comportamento reológico que irá representar o comportamento mecânico quando em processo de deformação devido a um campo de tensões. Esses conhecimentos servem para o desenvolvimento de produtos e correlações de parâmetros físicos e sensoriais ${ }^{4}$. A FME quando dispersa em um meio aquoso pode se comportar como fluido newtoniano ou apresentar características pseudoplásticas descritas por equações newtoniana ou não-newtoniana ${ }^{5}$ e este comportamento depende de vários fatores como a concentração de solutos, do $\mathrm{pH}$ do meio aquoso, da temperatura, do cisalhamento aplicado e entre outros fatores. Este trabalho foi realizado com o objetivo de se avaliar o comportamento reológico de uma farinha mista extrusada de bagaço de jabuticaba e arroz polido em um viscosímetro rotacional nas temperaturas de $25,30,40$ e $50^{\circ} \mathrm{C}$.

\section{Material e Métodos}

Foi utilizada como matéria-prima uma farinha mista extrusada de bagaço de jabuticaba e arroz polido produzida em trabalhos anteriores por ASCHERI et al. ${ }^{6}$ em condições de: proporção bagaço/arroz de 20/80 (p/p), velocidade do parafuso de $66 \mathrm{rpm}$ e temperaturas da $1^{\circ}$, $2^{\circ}$ e $3^{\circ}$ zona de aquecimento do extrusor de 50, 100 e $150^{\circ} \mathrm{C}$, respectivamente.

As medidas reológicas de uma suspensão da FME em água destilada (18/250, p/v) foram obtidas de acordo com a metodologia descrita no manual da Brookfield $\mathrm{DV}-\mathrm{II}^{+7}$ nas temperaturas de $25-50^{\circ} \mathrm{C}$, controladas em banho termostático com circulação de água. Os dados experimentais, em unidades de torque nas velocidades de $2,5,10,20$ e $50 \mathrm{rpm}$ (correspondentes a uma faixa de deformação de 2,64-66,0 $\mathrm{s}^{-}$), a partir de um volume de $8 \mathrm{~mL}$ da suspensão, foram obtidos utilizando-se um viscosímetro de cilindros concêntricos da marca Brookfield digital DV-II+ com adaptador de pequenas amostras e pino SC4-18. Os ensaios foram realizados em cinco repetições, sendo a tensão de cisalhamento resultante da média das repetições experimentais. A tensão de cisalhamento $(\tau)$ em pascal $(\mathrm{Pa})$ e a taxa de deformação $(\gamma)$ expressa no inverso do tempo $\left(\mathrm{s}^{-1}\right)$ foram calculadas de acordo com o manual do viscosímetro utilizado ${ }^{7}$ :

$$
\begin{gathered}
\gamma(\mathrm{s}-1)=\mathrm{RPM} \times \mathrm{SRC} \\
\tau(\mathrm{Pa})=\mathrm{TK} \times \mathrm{SMC} \times \mathrm{SRC} \times \text { Torque }
\end{gathered}
$$

Onde: $\mathrm{RPM}=$ velocidade do pino $(\mathrm{rpm}) ; \mathrm{TK}=$ torque constante do modelo de viscosímetro $(0,09373)$; SMC $=$ constante multiplicador do pino SC4-18 $(3,2)$; SRC = taxa de deformação constante do pino SC4-18 $(1,32)$; Torque $=$ torque $(\%)$ obtido pela medida de viscosidade.

$\mathrm{O}$ conjunto, $\tau \times \gamma$ foi ajustado, por regressão nãolinear, as equações de Newton, de Ostwald-de-Waelle (Lei da potência, O-W) e de Herschel-Bulkley (H-B), equações (3), (4) e (5), respectivamente.

$$
\begin{aligned}
& \tau=\eta \cdot \gamma \\
& \tau=\mathrm{K} \cdot \gamma^{\mathrm{n}} \\
& \tau=\tau_{0}+\mathrm{K}_{\mathrm{H} \cdot \gamma}{ }_{n}^{\mathrm{nH}}
\end{aligned}
$$

Onde: $\tau_{0}=$ tensão de cisalhamento inicial $(\mathrm{Pa}) ; \mathrm{K}$ e $\mathrm{KH}=$ índices de consistência (em Pa•sn e Pa.snH, respectivamente); $\eta=$ viscosidade newtoniana $(\mathrm{Pa}) ; \mathrm{n}$ e $\mathrm{nH}$ = índice de comportamento do fluido (adimensional).

A viscosidade de pasta (VP) foi determinada em um Analisador Rápido de Viscosidade (Rapid Visco Analyser, RVA da Newport Scientific, Austrália) seguindo-se basicamente a metodologia de materiais extrusados no manual do mesmo. Para a análise no RVA, $3 \mathrm{~g}$ de farinha extrusada com umidade corrigida para $14 \%$, em base úmida, e adicionou-se água destilada até peso final de $28 \mathrm{~g}$. A temperatura inicial de $25^{\circ} \mathrm{C}$ foi aumentada gradualmente a $95^{\circ} \mathrm{C}$ a uma taxa de aquecimento de $14^{\circ} \mathrm{C} /$ minuto, permanecendo constante nessa temperatura, por 
3 minutos. O resfriamento foi feito também gradualmente até atingir a temperatura final de $25^{\circ} \mathrm{C}$.

Para cada ajuste foi analisado o coeficiente de determinação $\left(\mathrm{R}^{2}\right)$ e o parâmetro qui-quadrado $\left(\chi^{2}\right) 9$ utilizando o programa gráfico ORIGIN 5.0 da Microcal Software, Inc. (1991-1997). O conjunto, $\tau \times \mathrm{x}$ foi ajustado, por regressão não-linear, as equações de Newton, de Ostwald-de-Waelle (Lei da potência, O-W) e de HerschelBulkley (H-B), equações (3), (4) e (5), respectivamente.

\section{Resultados e discussão}

Durante a experiência foi observado que as suspensões aquosas obtidas da FME foram pouco estáveis uma vez que os sólidos constituintes do bagaço que não sofreram modificações durante o processo de extrusão precipitaram logo após três minutos de cisalhamento. Com isto, para ter dados confiáveis, a leitura do torque, para todas as temperaturas analisadas, foi obtida após dois minutos de cisalhamento alcançando valores de desvio padrão e de coeficiente de variação satisfatórios (Tabela 1).

Na Tabela 2, têm-se os parâmetros dos três modelos utilizados para os ajustes dos dados experimentais e os respectivos índices de ajuste. Comparando-se os parâmetros estatísticos dos ajustes para os três modelos (Tabela 1), observa-se que o modelo de $\mathrm{H}-\mathrm{B}$ proporcionou os melhores ajustes em toda a faixa de temperatura estudada com $\mathrm{R}^{2}$ próximo a um e $\chi^{2}$ próximo a zero. Em seguida, o modelo da Lei da Potência apresentou os maiores $\mathrm{R}^{2}$ e por último o modelo de Newton, com valores de $\mathrm{R}^{2}$ menores. O parâmetro $\tau_{\mathrm{o}}$ do modelo de $\mathrm{H}-\mathrm{B}$ sofreu efeito da temperatura, diminuindo com o aumento desta com ligeiro aumento aos $50^{\circ} \mathrm{C}$. A viscosidade newtoniana e o índice de consistência da Equação (4) não correlacionaram com a variação da temperatura, porém, exibem uma tendência negativa com o aumento da temperatura até os $40^{\circ} \mathrm{C}$.

Quanto ao índice de consistência do modelo de H-B observou-se uma forte dependência da temperatura, crescendo $\mathrm{K}_{\mathrm{H}}$ linearmente com o aumento da temperatura (Figura 1). Os valores de $\mathrm{n}$ e $\mathrm{nH}$ (índice de comportamento de fluxo) para os modelos de O-W e H-B, vistos na Tabela 1 , foram todos menores que a unidade, indicando o comportamento pseudoplástico do fluido. Estes índices, embora indiquem o tipo de fluído, ambos apresentaram comportamento diferente frente à variação da temperatura. De maneira geral o valor de $\mathrm{n}$ correlacionou quadraticamente $\left(\mathrm{R}^{2}=0,975\right)$ exibindo um valor máximo entre as temperaturas de 30 e $40^{\circ} \mathrm{C}$, enquanto que, o valor de $\mathrm{nH}$ diminuiu exponencialmente em função da temperatura adequadamente representado pela equação de Arhenius com energia de ativação de $10,671 \mathrm{~kJ} / \mathrm{mol}$.

Tabela 1: Valores experimentais da tenso de cisalhamento $(\tau)$ de soluções aquosas de uma farinha mista extrusada de bagaço de jabuticaba e arroz, desvios padrão e coeficientes de variação $(\mathrm{CV})$ obtidos em diferentes torques e faixas de deformação $(\gamma)$ e temperaturas.

\begin{tabular}{|c|c|c|c|c|c|c|c|c|c|}
\hline \multirow{2}{*}{$\begin{array}{l}\text { Torque } \\
\text { (rpm) }\end{array}$} & \multirow{2}{*}{$\gamma\left(\mathbf{s}^{-1}\right)$} & \multicolumn{2}{|l|}{$25^{\circ} \mathrm{C}$} & \multicolumn{2}{|c|}{$30^{\circ} \mathrm{C}$} & \multicolumn{2}{|l|}{$40^{\circ} \mathrm{C}$} & \multicolumn{2}{|l|}{$\mathbf{5 0}^{\circ} \mathrm{C}$} \\
\hline & & $\tau(\mathbf{P a})$ & $\begin{array}{l}\text { CV } \\
(\%)\end{array}$ & $\tau(\mathbf{P a})$ & $\begin{array}{l}\text { CV } \\
(\%)\end{array}$ & $\tau(\mathbf{P a})$ & $\begin{array}{l}\text { CV } \\
(\%)\end{array}$ & $\tau(\mathbf{P a})$ & $\begin{array}{l}\text { CV } \\
\text { (\%) }\end{array}$ \\
\hline 2 & 2,64 & $6,39 \pm 0,14$ & 2,13 & $5,43 \pm 0,56$ & 10,33 & $3,94 \pm 0,07$ & 1,78 & $5,75 \pm 0,06$ & 1,07 \\
\hline 5 & 6,60 & $7,97 \pm 0,06$ & 0,74 & $7,12 \pm 0,58$ & 8,21 & $5,49 \pm 0,21$ & 3,78 & $7,54 \pm 0,20$ & 2,65 \\
\hline 10 & 13,20 & $9,92 \pm 0,15$ & 1,48 & $9,78 \pm 0,47$ & 4,77 & $7,96 \pm 0,21$ & 2,67 & $10,36 \pm 0,14$ & 1,40 \\
\hline 20 & 26,40 & $13,78 \pm 0,34$ & 2,50 & $14,07 \pm 0,43$ & 3,09 & $12,14 \pm 0,15$ & 1,27 & $14,30 \pm 0,26$ & 1,84 \\
\hline 50 & 66,00 & $25,40 \pm 0,27$ & 1,07 & $26,57 \pm 0,52$ & 1,95 & $20,98 \pm 0,21$ & 1,01 & $23,88 \pm 0,25$ & 1,06 \\
\hline
\end{tabular}


Tabela 2: Parâmetros dos modelos de Newton, de Oswald-de-Waelle e de Herschel-Bulkley para a farinha mista extrusada de bagaço de jabuticaba e arroz polido.

\begin{tabular}{crrrr}
\hline \multirow{2}{*}{ Parâmetro } & \multicolumn{5}{c}{ Temperatura $\left({ }^{\circ} \mathbf{C}\right)$} \\
\cline { 2 - 5 } & \multicolumn{5}{c}{$\mathbf{2 5}$} & $\mathbf{3 0}$ & $\mathbf{4 0}$ \\
\hline$\eta$ & 0,425 & 0,439 & 0,352 & 0,408 \\
$\mathrm{R}^{2}$ & 0,627 & 0,777 & 0,781 & 0,557 \\
$\chi^{2}$ & 21,648 & 15,976 & 10,297 & 22,963 \\
& \multicolumn{5}{c}{ Oswald-de-Waelle } \\
$\mathrm{K}$ & 2,811 & 2,268 & 1,826 & 2,987 \\
$\mathrm{n}$ & 0,518 & 0,582 & 0,582 & 0,492 \\
$\mathrm{R}^{2}$ & 0,971 & 0,986 & 0,994 & 0,993 \\
$\chi^{2}$ & 2,282 & 1,353 & 0,359 & 0,514 \\
& \multicolumn{4}{c}{ Herschel-Bulkley } \\
$\tau_{\mathrm{o}}$ & 5,582 & 4,136 & 2,275 & 3,472 \\
$\mathrm{~K}_{\mathrm{H}}$ & 0,371 & 0,575 & 0,811 & 1,149 \\
$\mathrm{n}_{\mathrm{H}}$ & 0,949 & 0,875 & 0,752 & 0,687 \\
$\mathrm{R}^{2}$ & 0,999 & 0,999 & 0,999 & 0,999 \\
$\chi^{2}$ & 0,026 & 0,022 & 0,044 & 0,019 \\
\hline
\end{tabular}

De acordo com o modelo de HB e Figura 1, quanto maior a temperatura maior é o comportamento pseudoplástico da farinha extrusada. Na Figura 2, têmse os pontos experimentais, representando as relações entre a tensão de cisalhamento e taxa de deformação, com as curvas de ajuste dos pontos pelo modelo de H-B. Verifica-se pela posição relativa das curvas, a redução nas tensões de cisalhamento com o aumento da temperatura, confirmando a pseudoplasticidade da suspensão aquosa de FME.

Este comportamento é melhor visualizado na Figura 3 , onde pode-se notar que a inclinação das curvas diminui com o aumento de da taxa de deformação. Isto evidencia uma diminuição da viscosidade aparente $\left(\eta_{\mathrm{a}}=\frac{\tau}{\gamma}\right)$ com o aumento de $\gamma$. Este fato se deve à orientação das moléculas na direção do escoamento e à quebra de agregados, que tornam a resistência ao movimento cada vez menor ${ }^{8}$. Ainda na Figura 3 se observa dois grupos de curvas sendo

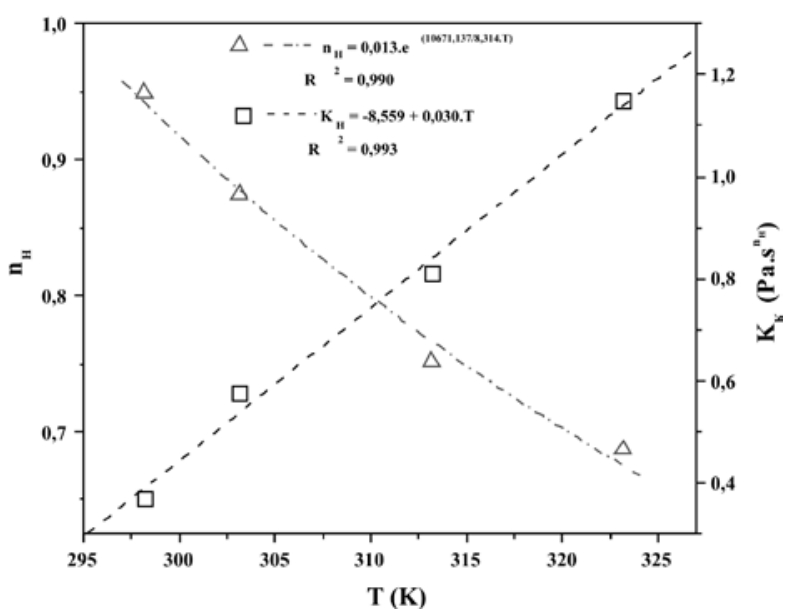

Figura 1: Efeito da temperatura sobre os parâmetros de índices de consistência e de comportamento do fluído da farinha mista extrusada de bagaço de jabuticaba e arroz.

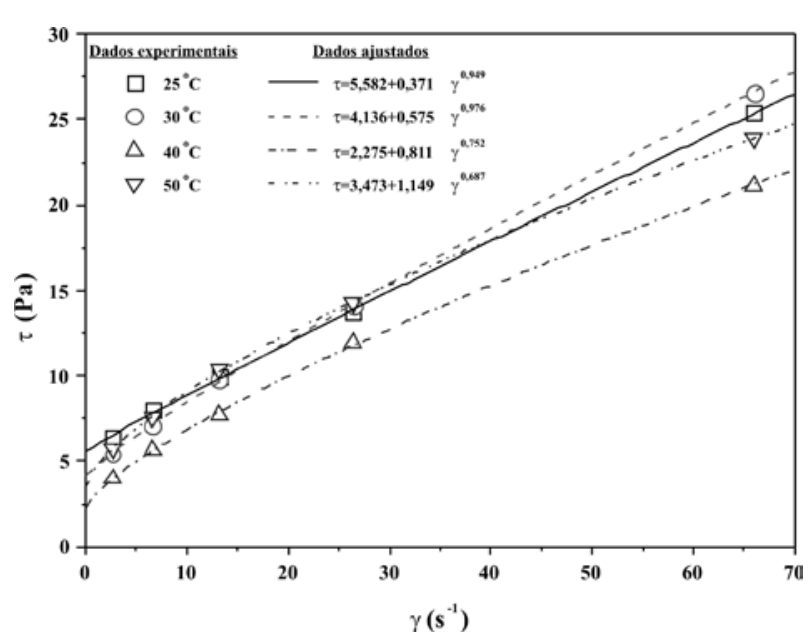

Figura 2: Relação entre tensão de cisalhamento $(\tau)$ e taxa de deformação $(\gamma)$ com ajuste pelo modelo de Herschel-Bulkley da suspensão aquosa da farinha mista extrusada de bagaço de jabuticaba e arroz.

que o primeiro está situada em alta viscosidade de 25, 30 e $50^{\circ} \mathrm{C}$ e o segundo situado em baixas viscosidades na temperatura de $40^{\circ} \mathrm{C}$.

Como o amido gelatinizado é o maior responsável pela viscosidade das pastas de produtos amiláceos4, os diferentes perfis de viscosidade provavelmente se devem à gelatinização total ou parcial do amido durante o processo de cocção dos grãos de arroz o que provocaria o aumento ou diminuição da viscosidade 


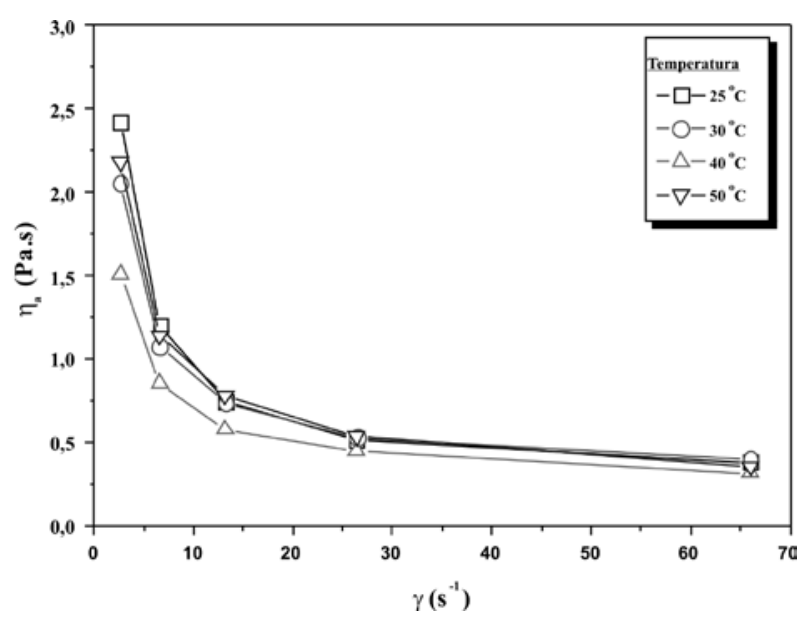

Figura 3: Curvas de viscosidade aparente ( $\eta$ a) em função da taxa de deformação $(\gamma)$ da suspensão aquosa da farinha mista extrusada de bagaço de jabuticaba e arroz, em diferentes temperaturas.

aparente. Para uma taxa de deformação constante de 6,60 s-1 se observa nitidamente a influência da temperatura na viscosidade aparente, com maior valor para a curva de $25^{\circ} \mathrm{C}$ e seguido pelas de 50,30 e $40^{\circ} \mathrm{C}$. Esta seqüência de viscosidade se deve a que a FME sofreu modificações na sua estrutura, principalmente no amido, gelatinizando-se durante o processo de extrusão pelo que fica mais solúvel e, portanto, aumenta sua viscosidade a temperaturas faixas, porém, este aumento é interrompido pelo aumento da temperatura (Figura 4). O aumento da viscosidade à temperatura de $50^{\circ} \mathrm{C}$ é explicado pela quebra remanescente de grânulos de amido que não sofreram modificações durante o processo de extrusão, estes incham absorvendo água e, conseqüentemente, aumentam a viscosidade. Este fenômeno pode ser visto na Figura 4, porém a uma temperatura próxima a $70^{\circ} \mathrm{C}$ onde se nota um pico de viscosidade.

\section{Conclusões}

O modelo de Ostwald-de-Waelle proporcionou um bom ajuste aos dados experimentais, porém, os melhores parâmetros estatísticos de ajuste foram obtidos com o modelo de Herschel-Bulkley. Os índices de comportamento de fluxo determinados através dos modelos reológicos de Ostwald-de-Walle e HerschelBulkley, apresentaram valores menores que a unidade,

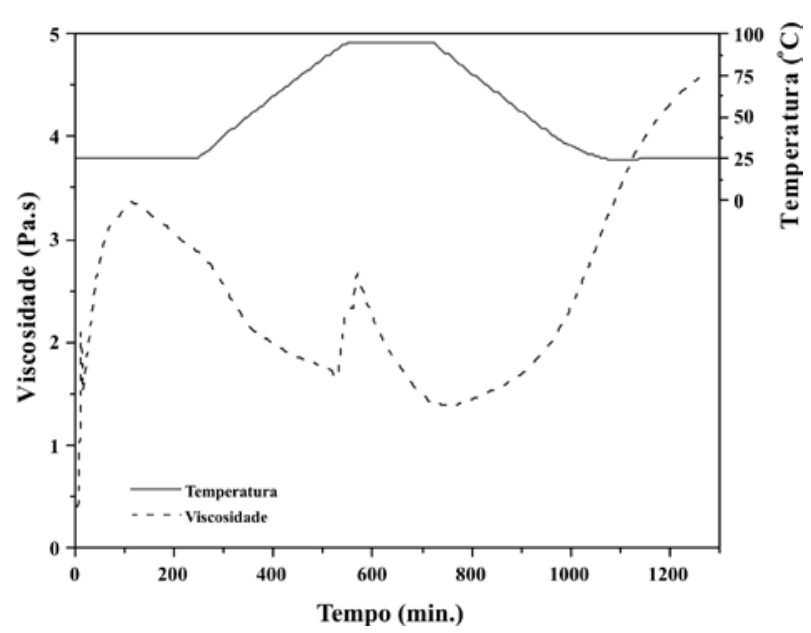

Figura 4: Perfil de viscosidade da farinha mista extrusada de bagaço de jabuticaba e arroz obtida através de RVA.

caracterizando a suspensão aquosa de farinha mista extrusada estudada como um fluido não-newtoniano, com características pseudoplásticas. Quanto aos parâmetros do modelo de Herschel-Bulkley, o valor do índice de consistência aumentou com o aumento de temperatura, e o índice de comportamento de fluxo diminuiu com o aquecimento cuja energia de ativação foi de $10,671 \mathrm{~kJ} /$ mol.

\section{Agradecimentos}

Os autores agradecem à Unidade Universitária de Ciências Exatas e Tecnológicas da Universidade Estadual de Goiás e ao Centro de Tecnologia de Alimentos da Empresa Brasileira de Pesquisa Agropecuária do Rio de Janeiro (CTAA/EMBRAPA - RJ) pelo suporte técnico.

\section{BIBLIOGRAFIA}

1. Asquieri, E.R.; Candido, M.A.; Damiani, C.; Assis, E.M. Fabricación de vino blanco y tinto de jabuticaba (Myrciaria jaboticaba Berg) utilizando la pulpa y la cáscara respectivamente. Alimentaria, 2004, 355, 97.

2. Asquieri, E.R.; Damiani, C.; Candido, M.A.; Assis, E.M. Vino de jabuticaba (Myrciaria cauliflora Berg): estúdio de las caracterísiticas físico-químicas y sensoriales de los vinos tintos seco y dulce, fabricados 
con la fruta integral. Alimentaria, 2004, 355, 111.

3. Ascheri, D.P.R.; Ascheri, J.L.R.; Carvalho, C.W.P. Caracterização da farinha de bagaço de jabuticaba e propriedades funcionais dos extrusados. Ciênc. Tecnol. Aliment. 2006b, 26, 897.

4. Bezerra, J.R.M.V. Comportamento reológico da polpa de manga (Mangifera indica L-Keitt). 2000, 159 Tese (Doutorado em Engenharia de Alimentos) Faculdade de Engenharia de Alimentos, Universidade Estadual de Campinas, Campinas-SP.

5. Ibarz, A.; Garvin, A.; Costa, J. Rheological behaviour of sloe (Prunus spinosa) fruit juices. Journal of Food Engineering. 1996, 27, 423.

6. Ascheri, D.P.R.; Andrade, C.T.; Carvalho, C.W.P.; Ascheri, J.L.R. Obtenção de farinhas mistas prégelatinizadas a partir de arroz e bagaço de jabuticaba: efeito das variáveis de extrusão nas propriedades de pasta. B. CEPPA. 2006a, 24, 115.

7. Brookfield DV-II+. Brookfield DV-II + programmable viscometer. Operating instructions manual $n^{\circ}$ M/97164. Disponível em: <www.brookfielengineering. com>. Acesso em: 24 nov. 2006.
8. Barnes, H.A.; Hutton, J.F.; Walters, K. An introduction to Rheology. Amsterdam: Elsevier Science Publishers, 1989.

9. Gomes, F. P. Curso de Estatística Experimental. 2th ed, São Paulo: ESALQ/USP, 2000.

\section{Diego P. R. Ascheri ${ }^{*}$, Pedro H. X. F. Silva', José L. R. Ascheri ${ }^{2}$ \& Carlos W. P. de Carvalho $^{2}$}

1 Unidade Universitária de Ciências Exatas e Tecnológicas da Universidade Estadual de Goiás, Anápolis/GO. CEP: 75001-970, Brasil.

2 Embrapa Agroindústria de Alimentos, Av. das américas 29501, Rio de Janeiro, CEP 23020-470. Brasil.

*E-mail: ascheridpr@gmail.com 